\title{
REVEALING THE NATURE OF THE HIGHLY OBSCURED GALACTIC SOURCE IGR J16318-4848 ${ }^{1}$
}

\author{
S. Chaty ${ }^{2,3}$ and P. Filliatre ${ }^{3,4}$
}

RESUMEN

La fuente de rayos X IGR J16318 - 4848 fue la primera fuente descubierta por INTEGRAL el 29 de enero. Llevamos a cabo observaciones en el óptico y el infrarrojo cercano (NIR) en el European South Observatory (ESO, La Silla) como parte de un programa "de oportunidad" (ToO). Descubrimos la contraparte óptica y confirmamos el candidato NIR que ya había sido propuesto. La espectroscopía NIR reveló una gran cantidad de líneas de emisión y perfiles P-Cygni. La distribución de la energía espectral de la fuente apunta hacia una luminosidad y temperatura altas con una absorción mayor que la absorción interestelar, pero dos órdenes de magnitud menor que la absorción de rayos X. Mostramos que la fuente es una Binaria de rayos X de Masa Alta (HMXB) a una distancia comprendida entre $\sim 1$ y $\sim 6 \mathrm{kpc}$, siendo el donador de masa una estrella de tipo temprano, probablemente una estrella sgB[e], rodeada por material circunestelar rico y absorbente. Esta sería la segunda HMXB con una estrella sgB[e] después de CI Cam en el caso de que una nueva clase de binarias de rayos X fuertemente absorbente esté siendo descubierta por INTEGRAL.

\section{ABSTRACT}

The X-ray source IGR J16318-4848 was the first source discovered by INTEGRAL on 2003, January 29. We carried out optical and near-infrared (NIR) observations at the European Southern Observatory (ESO La Silla) in the course of a Target of Opportunity (ToO) programme. We discovered the optical counterpart and confirmed an already proposed NIR candidate. NIR spectroscopy revealed a large amount of emission lines, including forbidden iron lines and P-Cygni profiles. The spectral energy distribution of the source points towards a high luminosity and a high temperature, with an absorption greater than the interstellar absorption, but two orders of magnitude lower than the X-ray absorption. We show that the source is an High Mass X-ray binary (HMXB) at a distance between $\sim 1$ and $\sim 6 \mathrm{kpc}$, the mass donor being an early-type star, probably a $\operatorname{sgB}[\mathrm{e}]$ star, surrounded by a rich and absorbing circumstellar material. This would make the second High Mass X-ray Binary (HMXB) with a sgB[e] star after CI Cam, indicating that a new class of strongly absorbed $\mathrm{X}$-ray binaries is being unveiled by INTEGRAL.

\section{Key Words: CIRCUMSTELLAR MATTER - STARS: EMISSION-LINE, BE - X-RAYS: BINARIES}

IGR J16318-4848 has been the first source to be discovered by the INTEGRAL imager IBIS/ISGRI on 2003 January $29,0.5^{\circ}$ south from the galactic equator (Courvoisier et al. 2003). A subsequent observation by XMM-Newton on 2003 February 10 localized it with an accuracy of $4^{\prime \prime}$, the high energy spectrum suggesting a high column density of $N_{h}>10^{24} \mathrm{~cm}^{-2}$ (Matt \& Guainazzi (2003), Walter et al. (2003)). In the course of a ToO programme at ESO to look for counterparts of high energy sources discovered by satellites including INTEGRAL (PI S. Chaty), we carried out on 2003,

\footnotetext{
${ }^{2}$ Université Paris 7, 2 place Jussieu, F-75 005 Paris, France. email: chaty@cea.fr

${ }^{3}$ Service d'Astrophysique, DSM/DAPNIA/SAp, CEA/Saclay, F-91 191 Gif-sur-Yvette, Cedex, France.

${ }^{4}$ Fédération de Recherche APC, Paris, France.

1 Based on observations collected at the European Southern Observatory, Chile (observing proposal ESO No $70 . \mathrm{D}-0340)$.
}

February, 23-25 photometric and spectroscopic observations in the optical and NIR of the high-energy source IGR J16318-4848, with EMMII and SOFI instruments on ESO/NTT. We discovered the optical counterpart and confirmed the NIR one (see Walter et al. (2003)) by independent astrometry. The optical/NIR images and spectra are shown in Figures 1. $2,3,4$ and 5 . We derived the absorption along the line of sight: $A v \sim 17.4$ magnitudes, and the temperature of the companion star: $\sim 18000 \mathrm{~K}$. The distance of the source is constrained between $\sim 1$ and $\sim 6 \mathrm{kpc}$. The $0.95 \mu \mathrm{m}-2.52 \mu \mathrm{m}$ NIR spectrum is highly unusual, very rich in emission lines. suggesting a highly complex and stratified circumstellar environment, or an enveloppe. Study of the spectral lines suggest a $\operatorname{sgB}[\mathrm{e}]$ star so the system would be a HMXB, probably hosting a neutron star. like CI Cam. The reader should refer to Filliatre $\&$ Chaty 

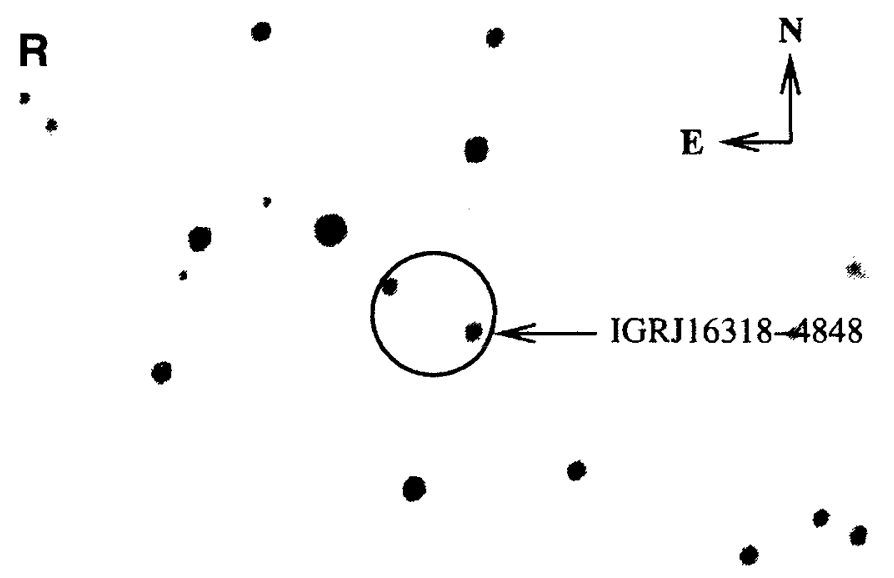

Fig. 1. R band image of the field of view of IGR J16318Kis. Wo reported the XMM moretainty circle of $4^{\prime \prime}$.

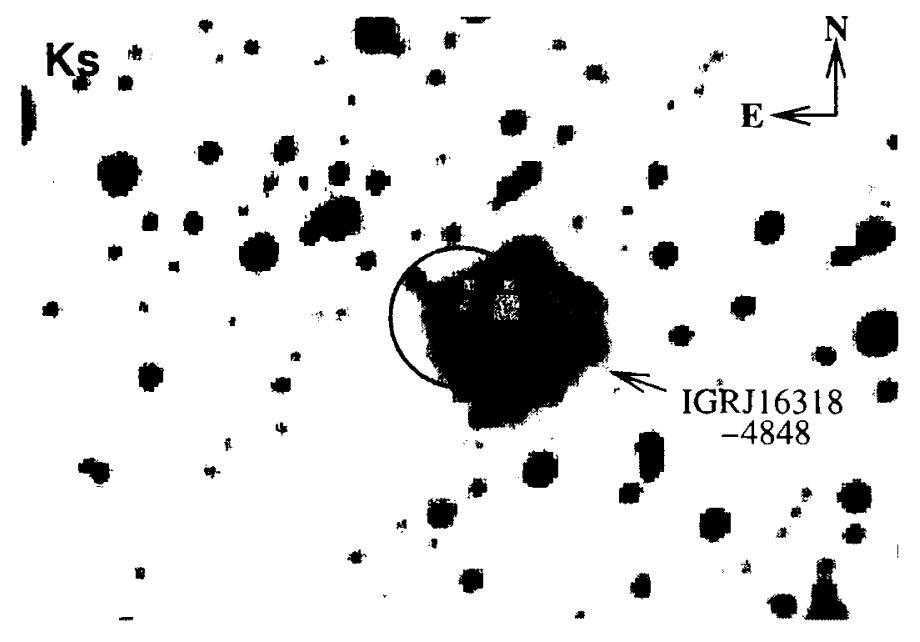

Fig. 2. Kis band image of the same field.

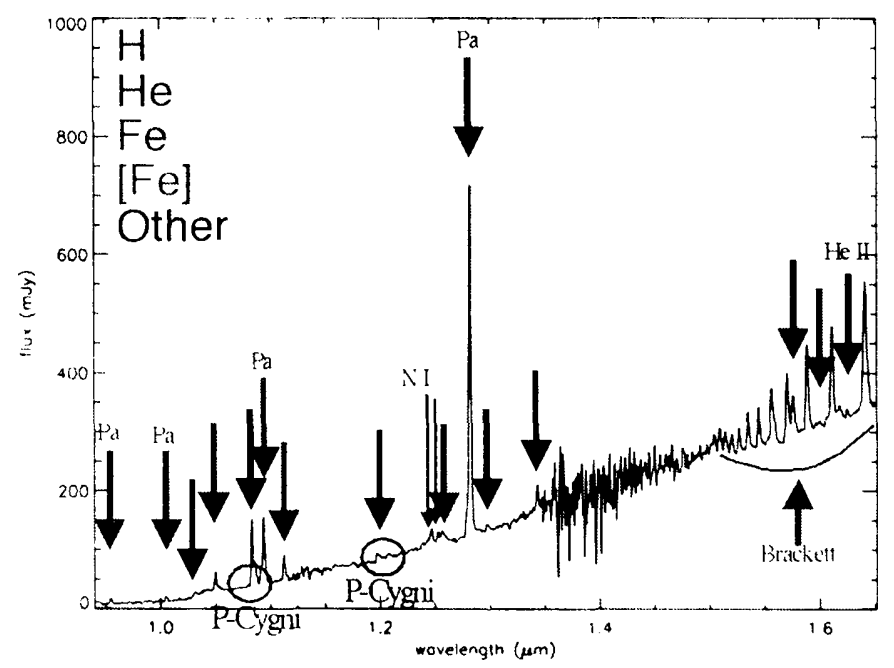

Fig. 3. NIR spectrum $(0.95-1.65 \mu \mathrm{m})$

(2004) for more details. INTEGRAL is on the course of revealing a new population of obscured high energy sources, which might help us to understand the evolution of high-energy binary sirstens.

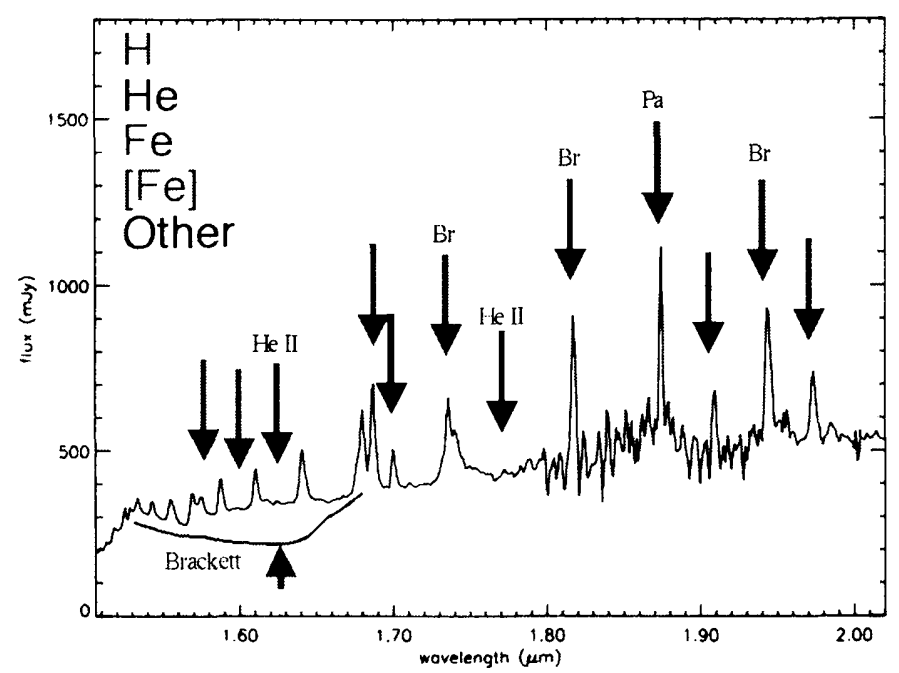

Fig. 4. NIR spectrum $(1.5-2.05 \mu \mathrm{m})$

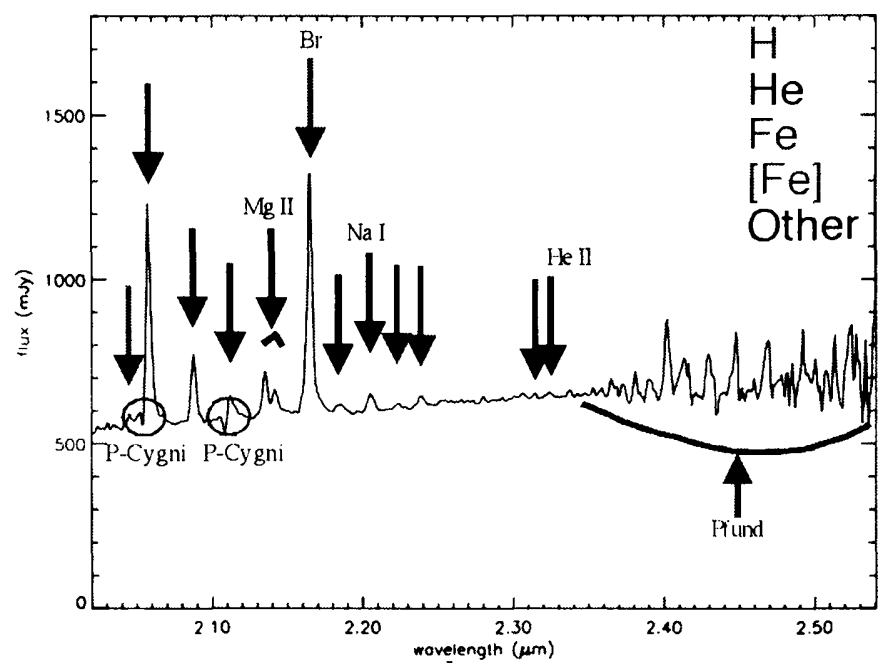

Fig. 5. NIR spectrum $(2.0-2.55 \mu \mathrm{m})$

$\mathrm{SC}$ is grateful to Ignacio Negueruela, Rob Hynes and Marc Ribó for very helpful discussions on the nature of the source during this IAU colloquium.

\section{REFERENCES}

Courvoisier, T., Beckmann, V., Bourban, G. et al. 2003, IAU Circ.., 8063

Filliatre, P., Chaty, S., 2004, in prep.

Matt, G. \& Guainazzi, M. 2003, MNRAS, 341, L13

Walter, R. et al. 2003, A\&A, 411, L427 